Relations industrielles

Industrial Relations

\title{
La politique salariale et le cycle économique
}

\section{Yves Dubé}

Volume 24, numéro 1, 1969

URI : https://id.erudit.org/iderudit/027987ar

DOI : https://doi.org/10.7202/027987ar

Aller au sommaire du numéro

\section{Éditeur(s)}

Département des relations industrielles de l'Université Laval

ISSN

0034-379X (imprimé)

1703-8138 (numérique)

Découvrir la revue

\section{Citer cet article}

Dubé, Y. (1969). La politique salariale et le cycle économique. Relations

industrielles / Industrial Relations, 24(1), 70-82. https://doi.org/10.7202/027987ar
Résumé de l'article

Dans cet exposé, l'auteur explique comment il ne croit pas plus à la politique salariale qu'il peut croire à l'inévitabilité du cycle économique. Il croit encore moins à la possibilité d'influencer le cycle économique par une quelconque politique salariale.
Tous droits réservés @ Département des relations industrielles de l'Université Laval, 1969
Ce document est protégé par la loi sur le droit d'auteur. L’utilisation des services d’Érudit (y compris la reproduction) est assujettie à sa politique d'utilisation que vous pouvez consulter en ligne.

https://apropos.erudit.org/fr/usagers/politique-dutilisation/ 
L'ensemble de ces programmes sociaux ont comme objet principal soit la réduction des privations de l'individu et de sa famille, soit la réduction de l'insécurité économique et sociale. Ils pourront avoir comme effet de situer l'individu dans un «univers d'aspirations légitimes > à l'intérieur duquel se situe l'optimum de bien-être. Le niveau optimum de bien-être sera fonction en dernier ressort du niveau des ressources disponibles et de certains choix quant à l'allocation de ces ressources. Dans l'optique d'une optimisation de la fonction de bien-être, l'allocation devra répondre aux ordres de préférence individuels et collectifs.

\section{Conclusion générale}

Il faudrait, en dernier ressort, discuter des exigences théoriques d'une politique globale, cohérente de sécurité sociale, de la nature de l'intervention étatique dans ce domaine et des freins structurels qui ralentissent son action.

\section{La politique salariale et le cycle économique}

\section{Yves Dubé}

Dans cet exposé, l'auteur explique comment il ne croit pas plus à la politique salariale qu'il peut croire à l'inévitabilité du cycle économique. Il croit encore moins à la possibilité d'influencer le cycle économique par une quelconque politique salariale.

Le titre de ce travail semble suggérer qu'il doit y avoir une politique salariale et que celle-ci doit être définie en tenant compte du cycle économique. C'est ce que plusieurs prétendent. Mais notre thèse sera tout autre. En fait, nous ne croyons pas plus à la politique salariale que nous pouvons croire à l'inévitabilité du cycle économique. Nous croyons encore moins à la possibilité d'influencer le cycle économique par une quelconque politique salariale.

Pour démontrer notre thèse nous tenterons d'examiner ce qu'a été au cours de la présente décen-

DUBE, Yves, doyen, Faculté des sciences sociales, professeur, Département d'économique, Université Laval, QUEBEC. 
nie l'expérience canadienne en matières de salaires. En effet, si l'on n'examine pas de près les événements tels qu'ils se sont produits, l'on sera porté à conclure à partir des événements de cette année ou de l'an passé, dans un secteur qui nous intéresse de plus près, qu'il faut des contrôles et que les gouvernements devraient intervenir pour limiter ou prévenir, selon les cas, les dégâts et même dicter la ligne de conduite à suivre en matière salariale. La définition d'une politique salariale peut être aussi vague que cela. Nous excluons ici naturellement la politique que le gouvernement suit à l'égard de ses propres employés.

\section{Qu'est-ce qui s'est véritablement passé-?}

On se souvient que pendant les années qui ont suivi la guerre jusqu'à 1956-57, la grande crainte de la plupart des économistes fut celle du chômage. Les discours et rapports de M. Coyne, alors Gouverneur de la Banque du Canada, vinrent à ce moment éveiller la population aux dangers de l'inflation laquelle croyait-il; devenait de plus en plus menacante. Les économistes, pour leur part, prétendaient que ce qui devenait un danger à ce moment n'était pas l'inflation mais au contraire le chômage. Au fait, la politique monétaire exposée et suivie par M. Coyne avait pour effet, d'après les économistes, d'augmenter le chômage et de compromettre la croissance économique du pays. Le Canada suivait d'ailleurs à cette époque des politiques semblables à celles qui étaient suivies aux États-Unis et qui ne contribuaient pas davantage à la prospérité canadienne.

A la suite du départ de $M$. Coyne et de la dévaluation du dollar canadien en 1962, il s'amorca une période de reprise pendant laquelle on parvint à résorber le chômage et même à abaisser le taux du chômage à $3.0 \%$, limite correspondant au plein emploi.

Pour bien saisir ce qui arrivé au cours de la période, il faut se référer aux statistiques sur les salaires, les profits, le chômage et les prix. Nous donnons ces statistiques aux tableaux I et II.

Disons au départ que pendant toute cette période, la main-d'oeuvre canadienne a augmenté à un rythme sans précédent et que le gouvernement devait avoir recours à une politique expansionniste, non seulement pour absorber la main-d'oeuvre additionnelle mais également résorber le chômage. Notons qu'au début de la période, le chômage était de $7.1 \%$ et que le taux d'augmentation de la main-d'oeuvre est passé de 1.5 à $4.0 \%$ au cours de la période. Il n'est donc pas étonnant que la production et l'emploi, dans ce contexte d'expansion économique, aient augmenté rapidement. 


\section{TABLEAU I}

Production nationale brute, salaires et traitements

\section{bénéfices des sociétés avant impôts}

(1960-67)

(millions de dollars et pourcentage d'augmentation par rapport à l'année précédente)

\begin{tabular}{|c|c|c|c|r|r|r|}
\hline & \multicolumn{2}{|c|}{$\begin{array}{c}\text { Production } \\
\text { nationale brute }\end{array}$} & \multicolumn{2}{c|}{$\begin{array}{c}\text { Salaires et } \\
\text { Traitements }\end{array}$} & \multicolumn{2}{c|}{$\begin{array}{c}\text { Bénéfices des sociétés } \\
\text { avant }\end{array}$} \\
\cline { 2 - 7 } & $\$$ & $\%$ & $\$$ & $\%$ & $\$$ & $\%$ \\
1960 & $36,287$. & & $18,245$. & & $3,338$. & \\
1961 & $37,471$. & 3.2 & $18,996$. & 4.1 & $3,427$. & 2.6 \\
1962 & $40,575$. & 8.3 & $20,233$. & 6.5 & $3,819$. & 11.4 \\
1963 & $43,424$. & 7.0 & $21,547$. & 6.5 & $4,188$. & 9.6 \\
1964 & $47,393$. & 9.1 & $23,433$. & 8.7 & $4,819$. & 15.0 \\
1965 & $52,203$. & 10.1 & $26,179$. & 11.7 & $5,199$. & 7.8 \\
1966 & $58,120$. & 11.3 & $29,661$. & 13.3 & $5,145$. & -1.0 \\
1967 & $62,068$. & 6.8 & $32,389$. & 9.1 & $5,020$. & -2.4 \\
\hline
\end{tabular}

Source: Comptes nationaux, B.F.S., Ottawa.

\section{TABLEAU II}

Chômage en pourcentage de la main-d'oeuvre

Indice des prix à la consommation

Augmentation de la productivité de la main-d'oeuvre

(1960-67)

\begin{tabular}{|c|c|c|c|}
\hline & $\begin{array}{c}\text { Chômage en } \\
\text { pourcentage de la } \\
\text { main-d'oeuvre }\end{array}$ & $\begin{array}{c}\text { Indice des prix } \\
\text { à consommation } \\
1949=100\end{array}$ & $\begin{array}{c}\text { Augmentation de la } \\
\text { productivité par } \\
\text { personne employée } \\
\text { par rapport à l'année } \\
\text { précédente }\end{array}$ \\
\hline 1961 & 7.1 & 129.2 & \\
1962 & 5.9 & 130.7 & 2.1 \\
1963 & 5.5 & 133.0 & 2.3 \\
1964 & 4.7 & 135.4 & 2.7 \\
1965 & 3.9 & 138.7 & 1.8 \\
1966 & 3.6 & 143.9 & 0.8 \\
1967 & 4.1 & 149.9 & 0.0 \\
\hline
\end{tabular}

Source: Quatrième exposé annuel du Conseil économique du Canada: L'Economie canadienne des années 1960 aux années 1970. Ottawa, 1967. 
Ce qui compte cependant, pour nos fins, est l'évolution relative des profits, des salaires, de la production et des prix. Nous pouvons constater qu'au début de la période, soit en 1962, 1963 et 1964, l'augmentation des bénéfices avant comme après impôt est très substantielle. L'augmentation se situe entre 9 et $13 \%$ avant impôt et entre 10 et $16 \%$ après impôt. La productivité augmente elle aussi, mais pendant ce temps, la masse salariale elle n'augmente qu'au rythme de 6 à $8 \%$, une partie de cette augmentation étant expliquée par la résorbtion du chômage. Or, en 1965, s'amorce la reprise dans les salaires. En 1965 et en 1966, l'augmentation passe à 10 et à $11.2 \%$. Les salariés deviennent de plus en plus exigeants même aggressifs, et les employeurs à cause du climat économique favorable et sans doute aussi grâce aux grandes liquidités accumulées aux cours des années précédentes, ne résistent guère. Les bénéfices des sociétés au cours de l'année 1965 augmentent alors à un rythme ralenti et en 1966 et 1967 vont même jusqu'à accuser une diminution. La diminution des profits s'accompagne d'une diminution dans le taux de changement de la productivité et, en 1967, l'augmentation du taux de croissance de la productivité devient nulle. La baisse des bénéfices des sociétés peut donc s'expliquer en partie par l'augmentation considérable des salaires et par la diminution du taux de changement de la productivité, lequel tombe à 0 .

Pendant ce temps, les pressions inflationnistes deviennent de plus en plus fortes. L'indice des prix à la consommation qui avait affiché une hausse de l'ordre de 2.5 points en 1963 et en 1964, augmente de 3.3 points en 1955, 5.2 points en 1966 et 5.1 points en 1967. Le taux de chômage augmente également entre 1966 et 1967 de 3.6 à $4.1 \%$, ce qui, toute légère soit-elle, représente la première augmentation depuis 1962.

Remarquons, au passage, que les divers facteurs mentionnés plus haut, ne semblent pas avoir évolué de la même façon aux États-Unis, ce qui fait que la conjoncture américaine a été bien différente de la nôtre. Il semble que les États-Unis viennent d'entrer dans la phase que nous avons traversé au cours des dernières années. La tendance à la hausse des salaires s'y est faite sentir plus tôt et la hausse s'est produite à une allure beaucoup plus régulière. Il en est de même de la productivité et des bénéfices des sociétés. L'inflation s'est faite sentir mais elle n'a pas pris l'ampleur que nous avons connue au Canada.

De cette analyse succinte, il faut retenir le climat de croissance économique extrêmement rapide dans un contexte économique expansionniste au départ, de capacité excédentaire et, à la fin, de surutilisation des ressources productives caractérisée par la présence de goulots d'étrangle- 
ment. Nous avons eu affaire, il semble, à un enchainement de phénomènes; l'augmentation de la demande effective entraînant une augmentation des bénéfices, cette dernière entraînant une augmentation des traitements. On a dû alors faire face progressivement à des goulots d'étranglement de diverse nature qui ont arrêté la croissance de la productivité. Dans ce contexte, il ne pouvait s'ensuivre qu'une augmentation des coûts, une accélération de la montée des prix et finalement la hausse du chômage.

Avant de conclure à la nécessité d'une politique salariale pour le Canada, il faut se demander si, après tout, cette évolution dans le contexte, n'était pas normale. Étant donné les conditions qui existaient au départ, les choses pouvaient difficilement se passer autrement. Que les salaires aient eu tendance à augmenter au début moins rapidement que les profits semble un phénomène normal dans un contexte d'abondance de maind'oeuvre. L'alternative aurait été de provoquer une augmentation plus rapide des salaires, ce qui aurait peut-être empêché la hausse subite que nous avons connue par la suite, mais qui, au départ, aurait peut-être freiné la croissance. Il ne faut pas oublier que le déclenchement de la croissance a été causé par la décision soudaine de la dévaluation et par une politique monétaire et fiscale plus expansive. On ne pouvait en même temps provoquer une reprise économique et les augmentations de salaires. La période qui avait précédé immédiatement en avait été une de stagnation dans les profits comme dans l'activité économique générale. L'objectif ne pouvait donc être que le retour le plus rapide possible au plein emploi quitte à ce que les pressions inflationnistes se fassent sentir. $\mathrm{Si}$ celles-ci se produisaient, on verrait bien à appliquer les politiques nécessaires. Pour l'avenir, il y aurait cependant lieu de prévoir. une évolution plus graduelle dans le temps et de poursuivre, au jour le jour, des politiques dans ce sens.

\section{Facteurs explicatifs de la montée des salaires}

Selon l'économiste américain G. Perry, (') l'évolution des salaires peut s'expliquer par les cinq facteurs suivants :

a) Le taux de profit des corporations au cours de la période précédente ;

b) Le taux de chômage dans la période précédente ;

c) Le niveau des prix ;

d) La direction du changement dans les profits;

e) la direction du changement dans le chômage.

(1) Unemployment, Money Wage Rates and Inflation, The M I T Press, 1966, pp. 19-34. 
Suivant Perry, ces différents facteurs expliqueraient presque $90 \%$ des augmentations de salaire qui se sont produites depuis la dernière guerre. D'année en année, l'un ou l'autre facteur devient prépondérant. Il semble bien qu'un modèle semblable pourrait servir à expliquer les hausses récentes de salaires au Canada.

On pourra comprendre, en se référant à un tel modèle, qu'au début de la période les augmentations de salaires ne pouvaient pas être considérables à cause des faibles marges de bénéfices et le haut niveau de chômage qui avait prévalu au cours des périodes précédentes. L'augmentation des bénéfices, la diminution du chômage et l'accélération dans la montée des prix devaient par la suite amorcer une augmentation plus rapide des salaires. La variable chômage joue alors un rôle plus important parce que l'effet d'une baisse donnée du chômage sur les salaires est beaucoup plus grand quand celui-ci est faible. La hausse des salaires entraîne finalement une baisse des bénéfices et une baisse d'autant plus grande que la productivité du travail baisse. La politique continuant quand même d'être expansionniste, les 'prix augmentent encore, mais l'action dé certains facteurs s'exerce maintenant en sens contraire et vient neutraliser l'action de ceux qui s'exercent encore à la hausse. Dans ce dernier stade, les hausses de salaires sont plus difficiles à justifier et les augmentations deviennent plus difficiles à obtenir d'employeurs qui ont tendance à résister davantage.

Si l'analyse est juste, on aurait été en mauvaise position pour justifier une politique salariale dans un contexte de déséquilibre complet dans les variables explicatives. Si l'on essaie d'établir un rythme normal de croissance des salaires, dans une année donnée, on risque soit d'être injuste à l'égard des ouvriers, soit de freiner la croissance. Mieux vaut donc essayer de jouer sur les grandes variables de l'économie plutôt que de s'attaquer spécifiquement à la politique salariale.

\section{Conséquence de l'augmentation des salaires et de la baisse de la productivité}

Dans les circonstances qui ont caractérisé les deux dernières années, soit l'augmentation considérable des salaires, l'augmentation des prix et le statu quo dans le domaine de la productivité, il n'y a pas de doute qu'une réaction drastique s'impose. En effet, l'on pourrait continuer à laisser les salaires et les prix augmenter mais on aurait tôt fait de s'apercevoir que ceci ne peut mener qu'à une inflation de plus en plus grande avec les difficultés que cela peut comporter au point de vue de la balance 
des paiements, de la stabilité du dollar canadien, sur la répartition du produit national brut entre ceux qui peuvent se protéger contre l'inflation et ceux qui ne le peuvent pas et même sur la production nationale ellemême. Effectivement une inflation qui se développe trop rapidement peut entraîner une baisse dans la production nationale. En effet, pourquoi les travailleurs accepteraient-ils de travailler à un certain salaire si un mois après ce salaire devait perdre une grande partie de sa valeur à cause de la montée des prix ? Pourquoi les marchands accepteraient-ils de vendre quoi que ce soit, si quelques temps après, ils devaient réaliser des bénéfices accrus pour avoir attendu ? Pourquoi les fournisseurs euxmêmes accepteraient-ils de vendre? Personne ne vendant, les prix continueraient de monter davantage et à la fin toute production serait arrêtée.

Il n'y a donc qu'une façon de contenir l'augmentation dans les prix tout en maintenant le plein emploi et assurant une augmentation dans les salaires, c'est d'essayer d'augmenter la productivité globale de l'économie. Mais c'est là l'enjeu.

L'employeur qui aura été en quelque sorte forcé d'accorder une augmentation de traitement plus forte que l'augmentation de la productivité à long ou à moyen terme de ses ouvriers aura tôt fait de modifier ses méthodes de production pour essayer de rétablir son niveau de profit à un niveau acceptable. S'il ne le fait pas, il sera forcé d'augmenter ses prix. Pour que ce dernier recours soit possible, il faut soit que l'entreprise soit dans une situation de monopole ou encore que toutes les firmes d'une même industrie aient été affectées par une augmentation semblable dans leurs coûts. Ceci a été le cas en ces dernières années des industries de service. Évidemment l'on suppose ici que l'État poursuit une politique de plein emploi.

Si l'entreprise affectée doit recourir à une modification de ses méthodes de production, elle songera à utiliser plus de machines par rapport à la main-d'oeuvre ou en ce qui concerne la main-d'oeuvre, une maind'oeuvre plus spécialisée. Il s'ensuivra naturellement pour cette entreprise des mises-à-pied. Ces mises-à-pied auront tôt fait de modifier les attitudes des ouvriers et des patrons quand ils se présenteront à la table des négociations, les premiers devenant moins exigeants dans leurs réclamations et les seconds étant en meilleure position pour s'opposer. Il y aura par la suite moins d'augmentations de coûts et donc moins d'augmentations de prix. 
Ainsi, il me semble que l'augmentation des salaires et des prix que nous avons connue au cours des dernières années est un phénomène cyclique, et que cette situation a déjà commencé à changer. Les politiques d'austérité pratiquées présentement par les gouvernements viendront renforcir les tendances déjà amorcées. Ce qui doit nous inquiéter surtout à l'heure actuelle, c'est que les correctifs introduits par les gouvernements soient venus trop tard et que conformément à ce qui se passe habituellement, ils soient non pas trop faibles, mais trop forts.

\section{Un cas particulier, celui des services publics}

Depuis un ou deux ans les employés des gouvernements et de certains services publics se sont vus accorder le droit de négocier des conventions collectives et même de recourir à la grève. Il faut reconnaître que ce droit est un droit maintenant acquis et que de toute façon il est difficile de refuser à un groupe d'employés le droit de faire la grève alors que d'autres l'ont. Il reste cependant que la négociation et le droit de grève dans les services publics posent certaines difficultés particulières. D'une part, il n'y a pas de prix ou de profits en fonction desquels on peut juger des bénéfices qui pourraient être accordés. Ce qui est plus, les coûts doivent être absorbés par tous les citoyens et sont payés sous formes de taxes. Si les augmentations de salaires sont trop fortes, ce qui arrive c'est qu'une partie de la population reçoit des paiements de transfert de la part de la collectivité en général pour la partie de l'augmentation qui ne correspond pas vraiment à une augmentation de services productifs rendus.

Ce qui est plus dangereux, c'est que les normes d'augmentation de salaire décidées pour la fonction publique en viennent à constituer une norme générale pour l'économie. Le fait que le gouvernement ou qu'un gouvernement ait accepté d'accorder de telles augmentations devient raison suffisante pour que les mêmes augmentations soient exigées dans le secteur privé. A cet égard, je ne saurais être trop d'accord avec la citation suivante que je tire du troisième exposé annuel du Conseil économique du Canada.

« 1) Dans tous les cas où le Gouvernement est l'employeur immédiat ou ultime, l'objectif de la politique doit être de maintenir un rapport aussi étroit que possible avec les salaires et autres formes de rémunération accordées par les bons employeurs du secteur privé; ce rapport doit être maintenu de façon courante, sans les retards excessifs et les remises au lendemain qui souvent se sont produits dans le passé et qui ont fini par engendrer 
le mécontentetement, par réduire l'efficacité et éventuellement par exiger des rajustements très considérables des salaires et traitements.

2) De temps à autre, à cause de l'établissement ou de l'expansion rapide de services particuliers, le Gouvernement peut se trouver dans l'obligation de recruter un grand nombre de fonctionnaires de certaines catégories ou de certaines disciplines particulières. Dans ce cas, il peut, comme tout autre employeur en pareilles circonstances, sentir le besoin de relever sensiblement les échelles de salaires applicables à ces catégories ou classes.

3) Lorsque le Gouvernement se croit dans l'obligation de faire des rajustements ou d'accorder des hausses très considérables de salaires en raison de besoins particuliers, pour remédier à certaines injustices, ou pour se rapprocher des normes du secteur privé, il est important qu'il en fasse connaître clairement les raisons de façon que son geste ne puisse pas être interprété comme une directive ou un modèle à suivre dans l'ensemble de l'économie. $\gg(2)$

Je crois que si ces normes étaient acceptées nous aurions en fait une sorte de politique de revenu pour le secteur public. Il ne faudrait pas s'attendre cependant à ce que les négociations se déroulent toujours dans la tranquilité. Il y aurait encore ici des heurts. On aurait au moins là une norme à long terme dont on ne pourrait trop s'éloigner sans qu'il se produise des distorsions majeures dans le régime des salaires.

\section{Politique salariale}

Ayant de cette façon fait le tour des problèmes qui se posent, j'aimerais enfin faire certaines observations sur ce que l'on peut appeler ici une politique salariale. Je prends cette expression dans son sens le plus général soit un effort soutenu de l'Etat pour contrôler des salaires dans les divers secteurs de l'économie.

J'espère avoir démontré plus haut que les fortes augmentations de salaires que nous avons connues depuis 3 ou 4 ans sont une conséquence directe du niveau très élevé et à la hausse des bénéfices qui ont prévalu, du niveau très bás du chômage, de la baisse gráduelle de ce dernier et de l'augmentation des prix.

Dans le cas d'au moins une de ces variables, le chômage, l'effet est absolument asymétrique en ce senș que'des bas taux de chômage sont asso-

(2) Troisième exposé annuel, Conseil économique du Canada, Ottawa, 1966, pp. 202 et 203. 
ciés à des rythmes d'augmentation de salaires beaucoup plus élevés que des taux plus élevés.

Nous admettons, en outre, qu'à long terme, il faut travailler à réaliser les cinq grands objectifs proposés par le Conseil économique, soit le plein emploi, une croissance économique rapide, une stabilité raisonnable des prix, une situation noble de la balance des paiements et une juste répartition des revenus croissants.

Or, précisément à cause des problèmes qui se sont posés dans le domaine des négociations collectives, ces cinq objectifs du conseil n'ont pu être atteints concurramment. Nous avons fait très bien un moment en ce qui touche aux prix, à un autre moment en ce qui touche à l'emploi. Notre balance des paiements a été en très bonne posture pendant toute la période.

Mais en ce qui regarde «la juste répartition des revenus croissants 》, le résultat a été loin d'être heureux. Comme tous les objectifs se tiennent, une faillite dans la réalisation de l'un d'entre eux compromet les chances de succès en ce qui concerne les autres. On n'a peut-être pas considéré ce problème assez sérieusement.

Il reste qu'à mon sens, il faut accepter cette situation comme inévitable et se dire que ce qui compte c'est que les objectifs soient réalisés à long terme. Mais il faut rester continuellement à l'affût et par des mesures de politique générale, viser à faire jouer les forces qui nous ramèneront à un équilibre à long terme entre les objectifs précités. Sinon nous devrons subir des distorsions qui iront en s'aggravant et ces distorsions engendront le désordre, lequel aura pour effet de compromettre le rendement de l'économie et le bien-être des citoyens.

La solution m'apparaît donc dans la voie d'une recherche d'une progression plus régulière dans les variables explicatives que nous avons examinées, laquelle dépend avant tout de la politique monétaire et fiscale.

\section{Examen des politiques proposées}

Les politiques qui ont été proposées jusqu'à maintenant pour «contrôler » l'augmentation des salaires ne me semblent donc pas justifiées parce qu'elles ne tiennent pas compte de la situation telle qu'elle se présente au Canada ou qu'elles ignorent certaines données fondamentales de l'économie canádienne. Mentionnons d'une façon spécifique a) la politique des revenus, b) la politique des « guidelines > et c) la dernière en liste, celle du Bureau de revision des salaires et des prix, établie par. M. Pearson. 


\section{a) Politique des Revenus}

Le Conseil économique a fait une étude systématique de la possibilité pour le Canada d'adopter une politique des revenus et s'est prononcé contre. Je ne peux faire à ce sujet que de citer le Conseil.

«Le Canada est loin de présenter le milieu idéal pour l'établissement d'une politique des revenus. Nous y trouvons non seulement de fortes disparités économiques régionales, mais aussi une répartition entre le fédéral et les provinces de la compétence législative dans quelques-uns des principaux secteurs compris dans les cadres d'une politique des revenus notamment les relations ouvrières:

Au Canada, les politiques publiques en matière de relations industrielles relèvent à la fois du gouvernement fédéral et des gouvernements provinciaux. A chacun toutefois appartient un champ d'action délimité par l'Acte de l'Amérique du Nord Britannique et les interprétations qu'en ont données les tribunaux. Dans les domaines des relations ouvrières, ces interprétations ont assigné le rôle principal aux provinces tout en laissant au gouvernement fédéral un secteur plutôt restreint mais difficile.

De ce fait, dans un large secteur de l'économie, le gouvernement fédéral n'aurait d'autre moyen que les appels à la bonne volonté pour faire observer l'aspect salaires d'une politique des revenus. (Evidemment, nous supposons ici une politique visant à la fois les prix et les salaires; une politique ne portant que sur un de ces éléments serait au départ politiquement et économiquement inacceptable). Dans des secteurs de l'économie aussi importante que celui de la fabrication, le Gouvernement n'aurait aucun droit formel d'intervenir dans les négociations et de chercher à faire conclure des ententes respectant les principes directeurs établis.

Sauf dans des cas d'urgence nationale, un état de guerre, par exemple, il manquerait au Canada l'un des principaux instruments requis pour la bonne application d'une politique des revenus. En effet, toute menace d'un recours à une réglementation directe des salaires et des prix serait absolument vaine. Le gouvernement fédéral n'a pas aujourd'hui le pouvoir d'imposer de telles mesures et n'a réussi à l'obtenir par les voies constitutionnelles qu'en période de guerre (en même temps qu'une compétence provisoire sur les relations ouvrières dans des secteurs qui normalement relèvent des provinces $\gg .{ }^{(3)}$

\section{b) LES 《 GUIDELINES *}

Comme le déclare le «Council of Economic Advisers 》 les «guidelines ou guideposts $>$ n'imposent à personne l'obligation de se restreindre dans ses demandes aux « guidelines \ proposés mais peuvent servir de gui-

(3) Ibid., pp. 177-178. 
des aux unions ouvrières et aux firmes ... Mais on ajoute que les « guideposts » ont pour but de «maintenir les augmentations de traitement à la croissance de la productivité dans l'ensemble de l'économie ».

Ces " guidelines 》 sont certes acceptables à long terme et pour l'ensemble de l'économie, mais ne sont d'aucune utilité en ce qui concerne les négociations dans un secteur particulier, à un moment donné du temps. Comme nous l'avons souligné plus tôt, il faut en outre tenir compte de l'évolution particulière des salaires au Canada par rapport à ce qui s'est produit aux États-Unis. Une firme particulière, en 1969, au Canada, devra faire face à des demandes de salaires qui dépendront de l'évolution des profits dans l'industrie de la région, du niveau précédent des salaires, du chômage, du niveau général des prix, ceci peu importe l'évolution des salaires ou de la productivité ailleurs ou dans l'économie dans son ensemble.

\section{c) Le Bureau de Revision des Prix et des salaires}

La dernière en liste, cette politique est aussi celle qui m'apparaît la moins justifiable. Tout le problème est d'une complexité telle que je ne puis voir ce qu'un tel Bureau viendrait faire. L'accent est mis sur la « revision ». Pour que le Bureau soit efficace, il devrait être en position de prévoir et essayer d'empêcher des développements de se produire, plutôt que de corriger ce qui s'est déjà produit. Je ne vois pas très bien quels sont les critères que l'on pourrait utiliser dans une aventure de ce genre. Une solution de ce genre est moins bonne que pas de solution du tout. Le marché peut faire de meilleures révisions.

\section{Conclusion}

La première conclusion qui se dégage de cette analyse est qu'il serait plus facile d'assurer une croissance plus régulière que celles que nous avons connues des salaires si l'économie pouvait toujours être maintenue au niveau correspondant au plein emploi. C'est parce que nous avons dû accroître la demande effective après la période de stagnation des années 1957-62 que les événements récents se sont produits. Le niveau de plein emploi ne peut être assuré que si l'on suit une politique monétaire appropriée c'est-à-dire absolument contraire à celle que l'on a suivi à la fin des années 50 .

La deuxième est qu'une politique de plein emploi doit être accompagnée d'une politique visant à augmenter la productivité des entreprises et de l'économie dans son ensemble. Autrement les augmentations de traite- 
ment résulteront en des augmentations de coûts de production et à la fin des prix. La main-d'oeuvre doit, en particulier, être constamment réentraînée et incitée à se déplacer des régions où des industries de productivité plus basse à celles qui le sont plus.

Ceci implique qu'une politique salariale au Canada n'est pas justifiée. Elle pourrait même être nuisible dans la mesure où elle introduirait des rigidités additionnelles. Mais la politique de laisser-faire à ce point de vue peut quand même s'accommoder de certains blocages de salaires et de prix dans des secteurs industriels ou des zones géographiques que l'on veut reconvertir. Mais même dans ces secteurs le blocage ne pourra être que temporaire.

Il y a toujours le danger que les parties en présence soient trop prudentes et interviennent trop peu et trop tard. C'est le cas des gouvernements et des unions ouvrières. Il se peut que la situation que nous avons connue ait pu être prévenue par une action plus éclairée des patrons, des unions et du gouvernement. Mais dans l'ensemble, étant donné les objectifs poursuivis, elle était inévitable.

\title{
Wage Policy
}

\author{
Jacques St-Laurent \\ Marc-Adélard Tremblay \\ Yves Dubé
}

\section{NOTION, OBJECTIVES, POSSIBILITIES}

There are many different definitions for the term \& wage policy s. In a broader sense it could be defined as being all policy influencing the development of wages. This description is much too broad for it includes all factors influencing the demand or supply of labor.

One could also speak about the wage policy of the employers (governments or others) and the wage policy of the union as an employer and as a representative organization of the workers.

Strictly speaking one might say that it regards the specific criteria or the e landmarks > established by a government in order to lead all employers or employees to be submitted to it. These are measures concerning the economy as a whole. 